\title{
Lag alternance in unidirectional coupled semiconductor lasers
}

\author{
J. Tiana ${ }^{1}$, J.H. Garcia-Lopez ${ }^{2}$, M.C. Torrent ${ }^{1}$, J. Garcia-Ojalvo $^{1}$ \\ 1. Universitat Politècnica de Catalunya, B.P. 08222, 11 C/Colom, E-08222 Terrassa, Spain \\ 2. Universidad de Gualajara CU Lagos, Enrique Diaz de Leon s/n, Paseos de la Montaña, Lagos de Moreno Jalisco, 47460 Mexico
}

Semiconductor lasers exhibit a wide range of dynamical behaviors such as low frequency fluctuations, coherence collapse, chaos induced by injection, etc [1]. Coupled semiconductor lasers are excellent devices to study the mechanisms leading to chaos synchronization [2]. In this presentation we show experimentally and numerically the existence of lag alternance in two semiconductor lasers coupled unidirectionally via two different paths (Fig.1a). The results obtained might shed light into how information is transmitted in a network where the nodes are linked via multiple paths.

A schematic diagram of the setup is shown in Fig.1a, where two semiconductor lasers (ML925B45F) are unidirectionally coupled, with LD1 being the emitter laser and LD2 being the receiver. LD1 oscillates chaotically due to the action of delayed feedback, while LD2 is coupled with LD1 via two different paths with two different delays. In order to control the relative influence of the two delay times we introduce an optical attenuator in one of the paths. Increasing the attenuation in path 1 , we are able to synchronize the lasers with either the lag-time of path $1\left(\tau_{1}=65 \mathrm{~ns}\right)$ or the lag-time of path $2\left(\tau_{2}=97 \mathrm{~ns}\right)$. The transition between these two extreme regimes corresponds to a region where the two paths are synchronized simultaneously. In order to distinguish which path is synchronized we calculate the cross-correlation function between time series of the two lasers. In Fig. $1 \mathrm{~b}$ we show the cross correlation function for three different values of the attenuation. Note that when the attenuation of the path 1 is low, the highest cross-correlation peak occurs at $\tau_{1}$ (path 1 is better synchronized than path 2), while when the attenuation is high the highest peak arises at $\tau_{2}$. When the attenuation corresponds to an intermediate value, the two paths are approximately equivalent and the value of the lag alternates randomly between $\tau_{1}$ and $\tau_{2}$. Numerical simulations of a generalized Lang-Kobayashi model reproduce the experimental results.
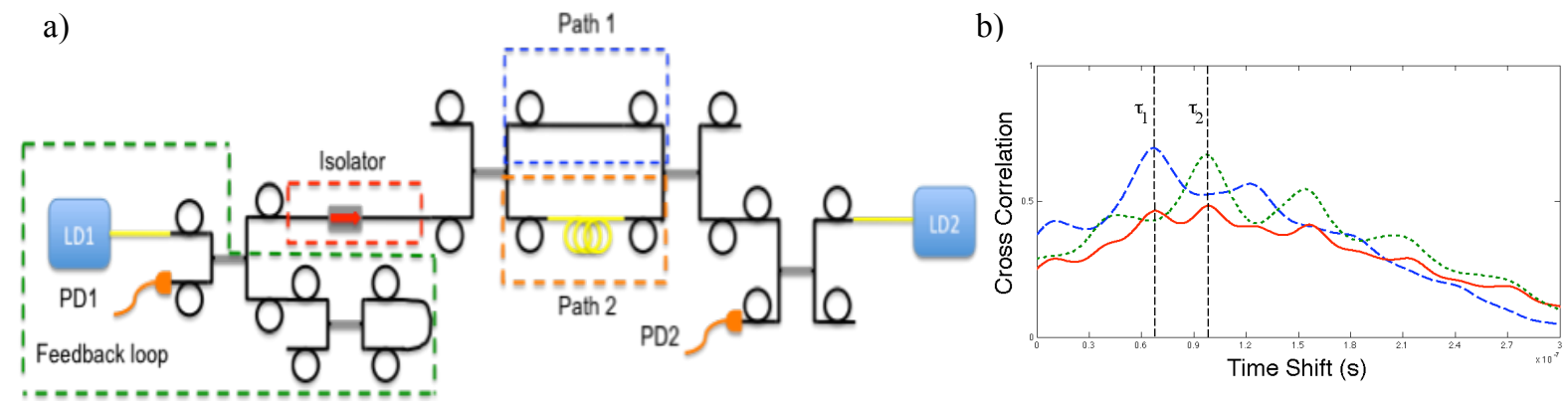

Fig. 1 a) Experimental setup of two unidirectionally coupled lasers through two lag times. PD1 and PD2 are the photodetectors. Note that all the setup is connected via optical fibres. b) Cross-correlation function between LD1 and LD2 for three different values of the attenuation in the path 1: $5 \mathrm{~dB}$ (blue dashed-line), $10 \mathrm{~dB}$ (red solid-line) and $15 \mathrm{~dB}$ (green dotted-line).

In order to understand how information is transmitted in the presence of multiple paths connecting two nodes in a network, we use again the setup presented in Fig.1a, with the emitter laser modulated by a periodic signal. We analyze the capacity of the receiver laser to filter a modulation introduced in the emitter laser, in the case where both delays dominate equally the dynamics (lag alternance). The experiments show that chaos-pass filtering occurs even in the case of lag alternance, which is necessary for any information to be transmitted down a system with multiple paths [3].

\section{References}

[1] J. Ohtsubo, "Chaotic dynamics in semiconductor lasers with optical feedback", Prog. Optics 44, 1 (2002).

[2] T. Heil, I. Fischer, W. Elsäser, J. Mulet and C.R. Mirasso "Chaos Synchronization and Spontaneous Symmetry-Breaking in Symmetrically Delay-Coupled Semiconductor Lasers ”, Phys. Rev. Lett. 85, 795 (2001).

[3] I. Fischer, Y. Liu, and P. Davis "Synchronization of chaotic semiconductor laser dynamics on subnanosecond time scales and its potential for chaos communication", Phys. Rev. A 62, 011801(R) (2000). 